\title{
Anti-tumor necrosis factor drug survival in axial spondyloarthritis is independent of the classification criteria
}

\author{
Pascal Zufferey $\cdot$ Jacqueline Ghosn • Fabio Becce • \\ Adrian Ciurea $\cdot$ Bérengère Aubry-Rozier $\cdot$ Axel Finckh • \\ Alexander K. So
}

Received: 10 April 2014 / Accepted: 9 July 2014 / Published online: 29 July 2014

(C) Springer-Verlag Berlin Heidelberg 2014

\begin{abstract}
To compare the impact of meeting specific classification criteria [modified New York (mNY), European Spondyloarthropathy Study Group (ESSG), and Assessment of SpondyloArthritis international Society (ASAS) criteria] on anti-tumor necrosis factor (anti-TNF) drug retention, and to determine predictive factors of better drug survival. All patients fulfilling the ESSG criteria for axial spondyloarthritis (SpA) with available data on the axial ASAS and $\mathrm{mNY}$ criteria, and who had received at least one anti-TNF treatment were retrospectively retrieved in a single academic institution in Switzerland. Drug retention was computed using survival analysis (Kaplan-Meier), adjusted for potential confounders. Of the 137 patients classified as having axial SpA using the ESSG criteria, 112 also met the ASAS axial SpA criteria, and 77 fulfilled the $\mathrm{mNY}$ criteria. Drug retention rates at 12 and 24 months for the first biologic therapy were not significantly different between
\end{abstract}

Electronic supplementary material The online version of this article (doi:10.1007/s00296-014-3094-z) contains supplementary material, which is available to authorized users.

P. Zufferey $(\bowtie) \cdot$ J. Ghosn · B. Aubry-Rozier · A. K. So Department of Rheumatology, Lausanne University Hospital, Avenue Pierre-Decker 4, 1011 Lausanne, Switzerland e-mail: zuffereypascal@gmail.com

F. Becce

Department of Diagnostic and Interventional Radiology,

Lausanne University Hospital, Lausanne, Switzerland

A. Ciurea

Department of Rheumatology, University Hospital Zurich,

Zurich, Switzerland

A. Finckh

Department of Rheumatology, University Hospital Geneva, Geneva, Switzerland the diagnostic groups. Only the small ASAS non-classified axial SpA group (25 patients) showed a nonsignificant trend toward shorter drug survival. Elevated CRP level, but not the presence of bone marrow edema on magnetic resonance imaging (MRI) scans, was associated with significantly better drug retention (OR 7.9, ICR 4-14). In this cohort, anti-TNF drug survival was independent of the classification criteria. Elevated CRP level, but not positive MRI, was associated with better drug retention.

Keywords Axial spondyloarthritis · Classification criteria $\cdot$ Anti-tumor necrosis factor $\cdot$ Drug survival

\section{Introduction}

The term spondyloarthritis $(\mathrm{SpA})$ covers various related disease entities with a common genetic background, as well as clinical and radiological similarities. Several sets of diagnostic and classification criteria have been proposed over time. Ankylosing spondylitis (AS) was defined by the Rome criteria and the modified New York (mNY) criteria since 1984. The European spondyloarthropathy study group (ESSG) proposed its own criteria in 1990. The latter criteria cover the entire spectrum of spondyloarthropathies, but appear to be less specific as some patients with chronic low back pain or peripheral inflammatory pain attributed to diffuse enthesitis may be falsely classified as SpA [1].

In 2009, the so-called Assessment of SpondyloArthritis international Society (ASAS) enacted new classification criteria for axial SpA [2, 3], which no longer requires radiographic joint damage on sacroiliac joints. The wider use of magnetic resonance imaging (MRI) confirms the presence of early inflammation on the sacroiliac joints in many patients before radiographic damage 
occurs. Thus, the ASAS criteria for axial SpA require several minor criteria associated with either the presence of HLA-B27 or a clear sacroiliitis on plain radiographs and/or on MRI [2, 4, 5].

The therapeutic armentarium of $\mathrm{SpA}$ and $\mathrm{AS}$ has increased considerably over the past decade [5]. Nonsteroidal anti-inflammatory drugs (NSAIDs) remain the first-line treatment of AS [6] but the anti-tumor necrosis factor-alpha (anti-TNF) therapies have proved to be very effective in AS patients. At first, they were only used in established long-lasting radiographic-proven AS, partially due to financial restrictions imposed by most national health authorities. Recent controlled randomized studies have suggested that they should be introduced at earlier stages of the disease, possible because of better identification by the newer ASAS and ESSG criteria [7]. Data from real-life settings evaluating the effectiveness of the new classification criteria on the response rates of biologic treatments are, however, quite rare [8].

Reimbursement of biological therapies in Switzerland is relatively liberal and without many administrative restrictions. The only requirement is clinical diagnosis of AS by a rheumatologist, without necessarily fulfilling $\mathrm{mNY}$ criteria as well as failing on conventional therapies. This has led to the prescription of anti-TNFs to patients diagnosed with SpA sometimes only based on the ESSG criteria. Many of these patients have non-radiographic SpA and some do not even fulfill the new ASAS definition of SpA [9].

The primary objective of this analysis was to compare anti-TNF drug survival [10] in patients fulfilling the mNY criteria versus the ESSG or ASAS criteria for axial SpA. The secondary objective was to look for predictive factors of better drug retention [11].

\section{Methods}

Study design

This is a retrospective observational cohort study of patients with axial SpA from a single University center in Switzerland (CHUV). The institutional ethics committee approved the study.

Patient selection

All the consecutive patients seen in our outpatients clinic between September 2011 and September 2012 with a diagnosis of SpA were susceptible to be included. We finally retained only those fulfilling the ESSG criteria, with sufficient data available to apply the ASAS criteria and who had received at least one biological treatment.
Classification definitions

Inflammatory back pain was defined according to the Berlin or Calin criteria $[12,13]$. ASAS positive (i.e., fulfilling the ASAS criteria of SpA) patients had to meet either the clinical arm (major criterion HLA-B27 positivity and at least two additional minor criteria), or the radiographic arm (definite sacroiliitis and at least one additional minor criterion). MRI was not mandatory to classify patients as ASAS positive if sacroiliitis was clearly apparent on radiographs (bilateral grade $\geq 2$ or unilateral grade $\geq 3$ ).

\section{Baseline clinical parameters}

Data were extracted from the patient's charts, into an electronic database. Variables collected included the clinical characteristics for the different SpA classifications, demographic parameters (age, sex), disease characteristics (duration of symptoms, disease duration, comorbidities) and treatment characteristics (initiation of biologic therapies, discontinuation of treatment). We also recorded measures of disease activity at initiation of biologic therapy (ESR, CRP, BASDAI, BASFI, VAS global activity).

\section{Follow-up and drug survival}

The survival of anti-TNF was computed based on the number of months of treatment for each individual patient. Treatment initiation was defined as the date of the first dose, and the date of discontinuation as the date of the first missed dose for interruptions of at least 3 months, or the date of switch to a second anti-TNF. We computed the median survival for the first antiTNF treatment and the percentage of patients still on drug at 12 and 24 months. We compared the proportion of "switchers" to alternative anti-TNF therapies in each group and looked for confounding factors and causes of discontinuation.

Discontinuation or switch of therapy was decided by the rheumatologist in charge of the patient based on clinical responses and not necessarily supported by strict measures of disease activity. The causes of discontinuation were categorized into four broad groups: adverse events, inefficacy or secondary loss of efficacy, remission or other. The latter category included miscellaneous causes for discontinuation in pregnancy, infection, surgery (considered a stop if the discontinuation lasted more than 3 months), poor compliance or patient decision. When available, the same disease activity parameters analyzed at initiation were also recorded at discontinuation of the biologic therapy.

\section{Radiographs and MRI}

All available radiographs and MRI of the sacroiliac joints at the time of biologic treatment initiation (within 6 months) 
were re-analyzed by an expert musculoskeletal radiologist blinded to the clinical history and previous radiographic reports. Sacroiliitis on MRI was defined on the basis of the ASAS criteria (one area of bone marrow edema visible on at least two sections, or two areas visible on one section, using fluid-sensitive (STIR or fat-suppressed T2-weighted) MRI sequences) [14]. When administered, contrast (gadolinium) enhancement was not taken into account for the diagnosis.

\section{Statistical analysis}

A comparison of baseline characteristics between the ESSG, ASAS and AS groups was performed using descriptive nonparametric methods. We used Kaplan-Meier plots, log-rank tests and multivariate Cox regression to analyze drug retention for biotherapy. We calculated the median anti-TNFs drug retention and the proportion still on biotherapies at 12 and 24 months. Based on previous literature, we adjusted the analysis for several potential confounders: sex, age at diagnosis, symptom duration at the initiation of biologic therapy, CRP level, MRI positivity, and type of anti-TNF. Hazard ratio (HR) for each potential cofactor with the interval confidence (IR) was calculated.

\section{Results}

Patients

Among the 226 patients who fulfilled the ESSG criteria, 137 were included according to the inclusion criteria (at least one anti-TNF treatment, enough data for axial ASAS calculation, MRI available at time of biologic treatment to ascertain the ASAS negative definition, follow-up of at least 1 year).

Radiographs and MRI of sacroiliac joints were available for 110 and 102 of the 137 patients, respectively. They were revised and reclassified by an expert radiologist according to the ASAS recommendations (see "Methods"). A discordant X-ray interpretation was found in $19 \%$, while discordance for MRI was noted in $15 \%$. Thirteen radiographs were changed from negative to positive, whereas eight were converted from positive to negative. For MRI, 13 cases were changed from negative to positive, whereas three cases were converted from positive to negative.

\section{SpA classification}

After revising the imaging data, 112 out of 137 patients meeting ESSG criteria also met the ASAS criteria for axial SpA. In this latter group (ASAS positive), 77 corresponded to AS according to the $\mathrm{mNY}$ definition (definite sacroiliitis on X-rays plus one minor criterion), while 35 were considered to have non-radiographic axial SpA (19 patients classified by the imaging arm and 16 patients by the clinical arm of the ASAS classification). Twenty-five ESSG positive patients were classified as ASAS negative patients because HLA-B27 was negative, and they had no sacroiliitis on radiographs and/or MRI.

\section{Baseline and demographic data}

Comparison of the baseline clinical and demographic data between ESSG positive, ASAS positive and AS positive patients is summarized in Table 1 . There were no statistically significant differences between ESSG positive, ASAS positive and AS positive SpA patients. In ASAS negative patients, the diagnosis was established much more recently (2009 vs. 2003), they were older at diagnosis (41 vs. 30 years) and had a shorter duration of symptoms (39 vs. 110 months). The proportion of patients with a positive MRI (101/137 available) and the presence of HLA-B27 (all HLA-B27 data available) in the different groups was significantly different, respectively: 59/46 \% in ESSG positive, $59 / 58 \%$ in ASAS positive, $78 / 78 \%$ in AS positive and $0 / 0 \%$ in ASAS negative patients (see Table 1).

Follow-up

Median drug survival for the first biologic therapy was not significantly different between the classification groups: ESSG positive, 11 (IR 6-15) months; ASAS positive, 12 months (IR 7-19); AS, 8 months (IR 6-13), respectively. Only ASAS negative patients showed a trend toward a lower survival: only 6 months (IR 4-14).

The retention rates at 12 and 24 months were also not significantly different: ESSG, 44 and $37 \%$; ASAS positive, 43 and $32 \%$; AS, 49 and $36 \%$, respectively. Once again, ASAS negative patients had a lower retention rate: 36 and $21 \%$. This was not statistically significant, however, because of the low numbers, but potentially clinically relevant. The survival curves did not diverge (Fig. 1) except again for the ASAS negative group after 1 year, but the number of patients still on medication in this group was quite small.

Mean disease activity objective parameters such as BASDAI, BASMI at baseline and at medication discontinuation were not significantly different between the various groups (Table 1). The mean BASDAI corresponded to values needed in many countries for the approval of biologic therapies in SpA by medical authorities: around or above 6. These data were, however, available at baseline and after follow-up in only half of the patients preventing reliable correlation with drug discontinuation. 
Table 1 Baseline data: clinical, biological and imaging features of the 137 patients treated with biologic therapies, according to the fulfillment of ASAS criteria or not

\begin{tabular}{|c|c|c|c|c|c|}
\hline & ESSG+ & ASAS+ & $\mathrm{AS}+$ & ASAS- & $p$ value \\
\hline Number of patients & 137 & 112 & 77 & 25 & - \\
\hline Gender: male/female & $60 / 77$ & $50 / 62$ & $35 / 42$ & $10 / 15$ & NS \\
\hline Mean (SD) age at first symptoms (in years) & 35.7 & $30.8(10.7)$ & $31.5(10.7)$ & $41.6(12.6)$ & $<0.001 *$ \\
\hline Mean (SD) age at diagnosis (in years) & 39.1 & $35.3(10.8)$ & $35.6(10.3)$ & $44.4(12.8)$ & $0.004 *$ \\
\hline Mean (SD) duration of symptoms (in months) & 132.6 & $164.8(126.6)$ & $164.2(121.0)$ & $77.4(63.2)$ & $<0.001 *$ \\
\hline Median year of diagnosis & 2005 & 2007 & 2004 & 2009 & - \\
\hline HLA-B27+ & $46 \%$ & $58 \%$ & $78 \%$ & $0 \%$ & $<0.001 *$ \\
\hline Inflammation of sacroiliac joints on MRI (101 available) & $\begin{array}{l}59 \% \\
60 / 101\end{array}$ & $\begin{array}{l}78 \% \\
60 / 76\end{array}$ & $\begin{array}{l}78 \% \\
39 / 50\end{array}$ & $\begin{array}{l}0 \% \\
0 / 25\end{array}$ & $<0.001 *$ \\
\hline Enthesitis & $53 \%$ & $51 \%$ & $46 \%$ & $44 \%$ & NS \\
\hline Recurrent or past uveitis & $21 \%$ & $25 \%$ & $27 \%$ & $8 \%$ & NS \\
\hline Inflammatory bowel disease (Crohn or RCUH) & $15 \%$ & $14 \%$ & $17 \%$ & $4 \%$ & NS \\
\hline Psoriasis & $8.9 \%$ & $4.5 \%$ & $5.2 \%$ & $12 \%$ & NS \\
\hline Positive family history (for AS, Psoriasis, IBD, uveitis, reactive arthritis) & $27 \%$ & $33 \%$ & $31 \%$ & $5.9 \%$ & $<0.05^{*}$ \\
\hline Systemic inflammation (CRP $>10 \mathrm{mg} / \mathrm{l})$ & $29 \%$ & $26 \%$ & $24 \%$ & $33 \%$ & NS \\
\hline Peripheral arthritis/synovitis & $55 \%$ & $45 \%$ & $28.6 \%$ & $60 \%$ & NS \\
\hline Mean (SD) BASDAI at baseline & $6.3(2.1)$ & $6.2(2.2)$ & $6.0(2.2)$ & $7.2(1.6)$ & NS \\
\hline Mean (SD) BASMI at baseline & & $5.2(2.3)$ & - & $5.8(1.9)$ & NS \\
\hline
\end{tabular}

* Significantly different between the ASAS- and ASAS +, ESSG + and AS + groups

Fig. 1 Kaplan-Meier survival curves at 1 year for biologics in ESSG positive, ASAS positive, ASAS negative and AS positive SpA patients at 12 and 24 months
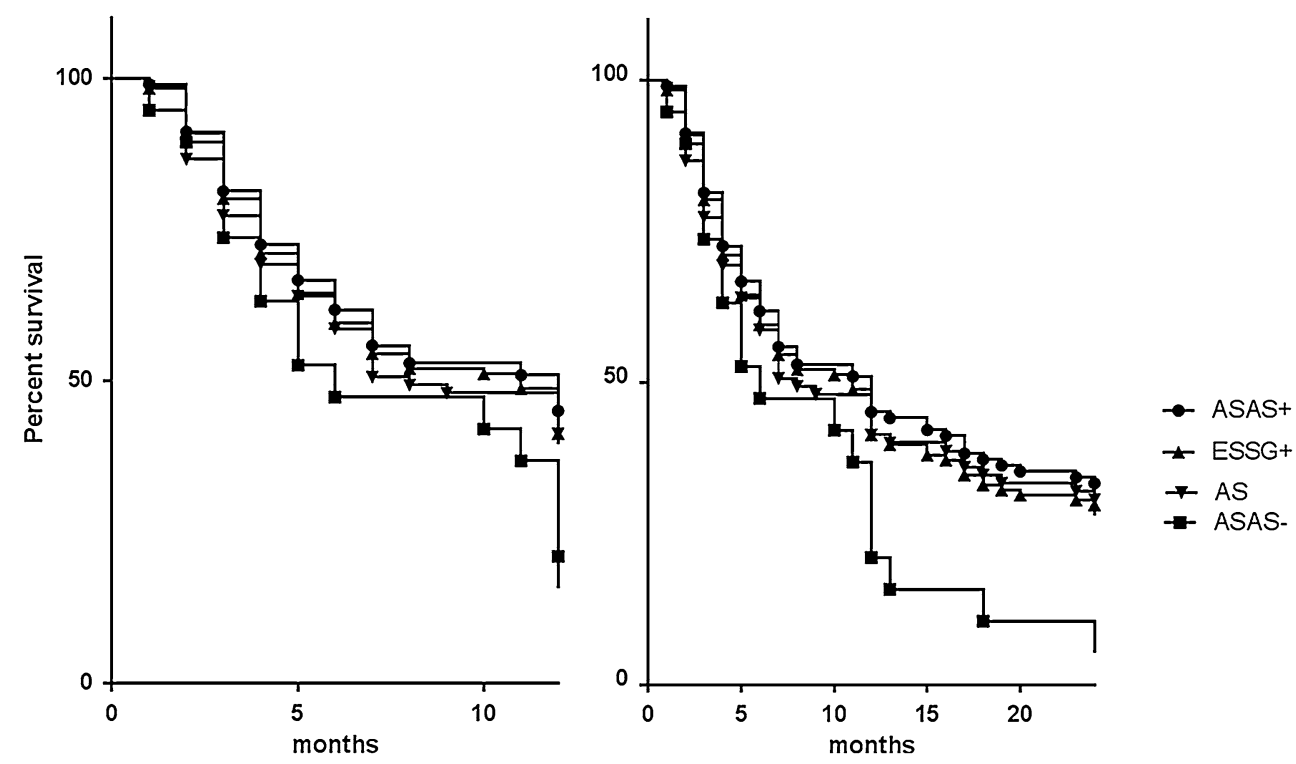

Confounders and predictors of survival

Only CRP came out as a significant overall predictor [15] of better survival in the drug survival analysis; (HR 7.9, IR $4-14, p<0.001$ ) for ESSG positive, ASAS positive and AS positive groups (Fig. 2). The numbers of patients with elevated CRP were too small in ASAS negative patients to be reliable. The median drug survival rate was 17.5 months for patients with elevated CRP and 8.0 months for patients with CRP below detection levels at inclusion. The difference was significant for all groups but particularly evident in the ASAS positive group with $61 \%$ survival at 12 months with an elevated CRP at inclusion compared to $37 \%$ survival with low CRP $(p=0.024)$.

No other predictors of better drug survival were apparent. The drug retention rates at 24 months were identical in patients with positive or negative MRIs for inflammation around sacroiliac joints [16]. The survival curves were 


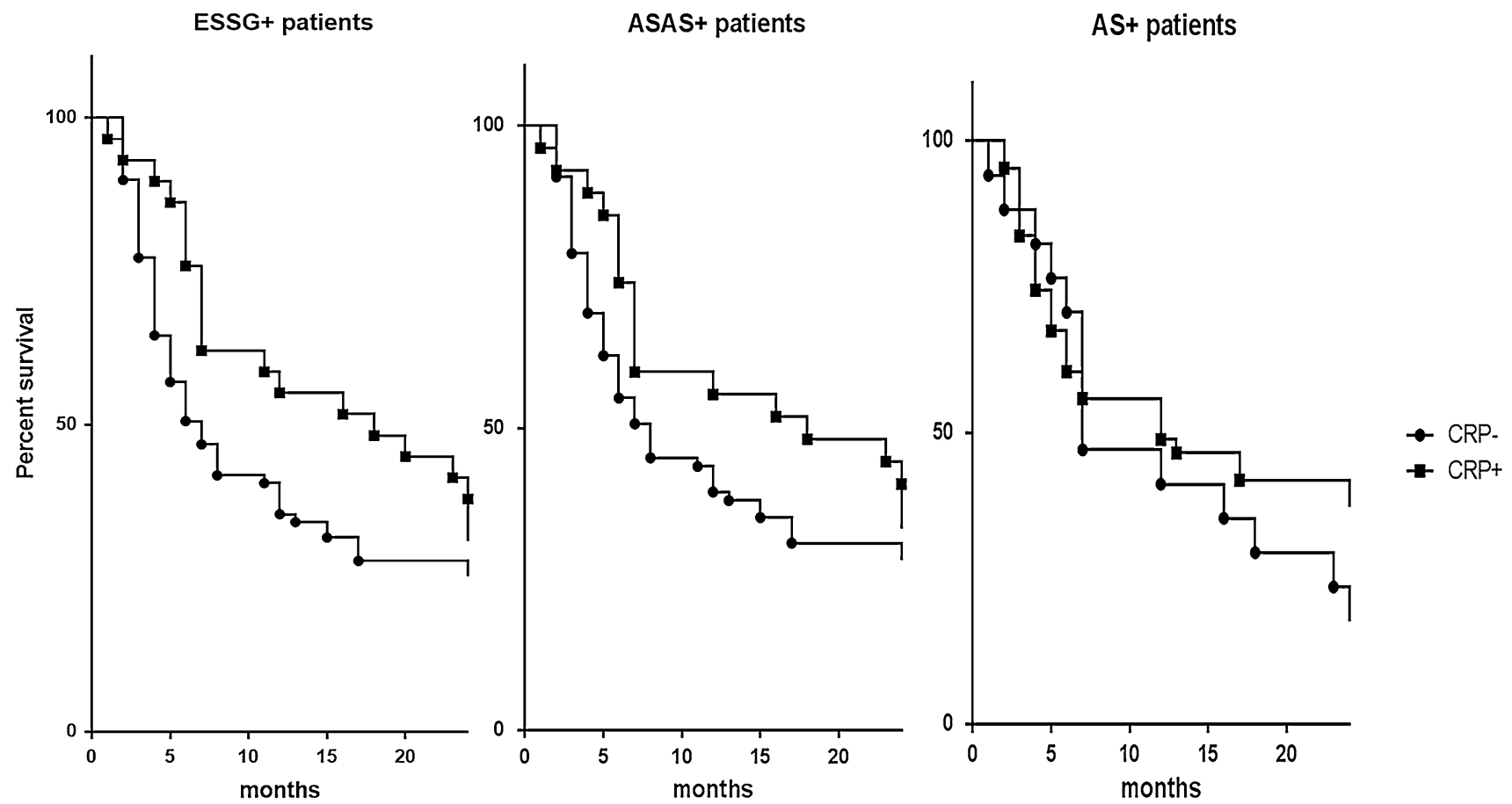

Fig. 2 Kaplan-Meier survival curves at 1 year for biologics according to CRP level at baseline in ESSG positive, ASAS positive and AS positive SpA patients. $(p<0.05$ for all groups)
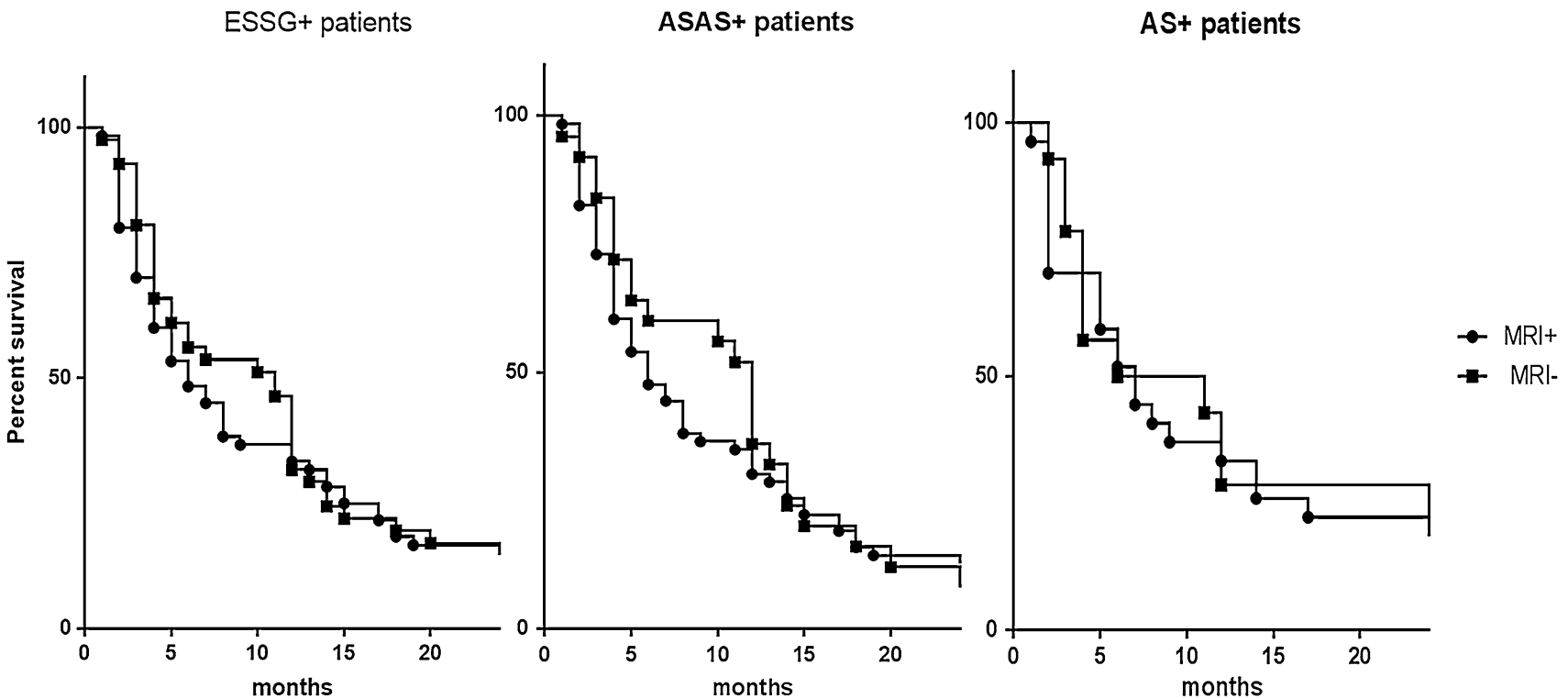

Fig. 3 Kaplan-Meier survival curves at 2 years depending on the presence or not of inflammation on MRI at introduction of biologics according to ESSG positive, ASAS positive and AS positive SpA patients

similar in all groups with positive or negative MRIs for inflammation on sacroiliac joints (Fig. 3).

Patients with longer symptom duration ( $>24$ months) had a drug survival rate of $48 \%$, while patients with shorter symptom duration ( $<24$ months) had $37 \%$ at 12 months (HR for longer duration 0.99, IR 0.6-1.5, $p=0.9$ ) [17]. Gender in contrast did not significantly influence drug survival (HR for male 0.76, IR 0.4-1.2, $p=0.2)$ [18].

We also found no differences in results between the four main TNF-blockers used (adalimumab $35 \%$; etanercept $31 \%$; infliximab $27 \%$; golimumab $5 \%$ ) after correcting 
for confounders such as sex, age, disease duration and beginning of drug treatment.

Similarly, excluding patients with drug discontinuation due to side effects $(23 \%)$ did not modify the survival curves in any of the groups (data available online).

\section{Discussion}

This monocentric retrospective "real-life" study suggests that anti-TNF drug retention does not depend on the criteria used to diagnose SpA. Indeed, drug survival was comparable regardless of the classification methods used; ESSG, ASAS, mNY. Only the small ASAS non-classified, ESSG classified patient group, negative for HLA-B27 and the absence of sacroiliitis on radiographs and MRI showed a nonsignificant trend toward shorter drug survival rates (Fig. 1). We suspect that at least some of these ASAS nonclassified patients did not have $\mathrm{SpA}$ and therefore presented primary resistance to anti-TNF therapy. The lack of difference in drug survival in ASAS and ESSG classifications and the AS defined group could be due to the fact that the groups are interrelated with many patients fulfilling all three classification criteria. Drug retention may be more influenced by specific characteristics such as: sex, duration of symptoms, baseline CRP and inflammation on MRI rather than global diagnostic classification.

Some studies have suggested that classification criteria select different populations of SpA patients [20]. In the present work we found no significant differences in the malefemale ratio, active enthesitis, inflammatory bowel disease, psoriasis, CRP levels, peripheral arthritis and disease activity according to the BASDAI between the different groups (Table 1).

However, the small patient group classified by ASAS as SpA-negative had some particularities. They were older at first symptoms and diagnosis, had shorter symptoms duration at the time of biologic treatment, were diagnosed more recently and less often showed a family history.

Shorter illness duration at time of diagnosis and introduction of biologic treatment in ASAS-SpA-negative and non-radiographic SpA patients confirming that diagnosis of $\mathrm{SpA}$ is usually made earlier in the course of disease in these patients has been observed in previous studies [20]. This could have some influence on the response to treatment. Nevertheless, according to the literature data, the definite benefit of early therapy on treatment response is still debated mainly because it is not so easy to reliably calculate the symptom duration in SpA. In the present work, no significant difference was found for the survival of the first biologic therapy according to symptom duration.

Several other predictors of better drug survival have been proposed in the literature. Elevated CRP at baseline has been shown to be the best one [15]. Our study confirms the importance of CRP. Indeed, among the criteria analyzed in this study: CRP levels ( $>$ or $\leq 10 \mathrm{mg} / \mathrm{l}$ ), gender (male or female), ASAS criteria (positive or negative) and type of anti-TNF therapy, only an abnormal CRP level at baseline came out as an overall significant predictor of better drug survival. The difference was significant whatever the classification used. It was, however, particularly evident in the ASAS classified patient group.

Inflammation visible on MRI scans can precede, sometimes by many years, the development of structural lesions visible on radiographs [16]. Positive MRIs have been shown to be a predictor of good response to biologic therapies in a few randomized studies [16]. However, real-life data on the predictive value of MRI for the response to biologic therapies in SpA are scarce. This is probably due to the fact that in most countries up to yet, biologic therapies are only used when diagnosis is confirmed on conventional radiographs. In these situations, there is no need and no yet-proven rationale to perform MRI. In our study, due to easy access to MRI in Switzerland, most of the patients had an MRI performed. The interpretation of MRI scans by a radiologist not specialized in musculoskeletal imaging can be hazardous [19]. This was confirmed by our results. We observed about $20 \%$ discordance between the initial interpretation of MRI and the one performed by an experienced musculoskeletal radiologist who strictly applied the diagnostic criteria established by the ASAS committee.

Even after revision of MRI data by an expert, we could not confirm that inflammation on MRI had significant influence on drug retention rate. Indeed, we found no significant difference in survival rates whether patients had a positive or negative MRI. This conclusion may be restricted by the fact that only inflammation around the sacroiliac joints and not the entire spine was taken into account. Moreover, the magnitude of inflammation was not analyzed in our patients.

This study has several strengths. The quality of data is relatively high for a real-life cohort; in particular, we had a high percentage of patients for which X-rays, HLA-B27 and even MRI were available. Even though the ASAS non-classified group was quite small, this is one rare study where TNF-blocker response was evaluated in such patients.

There are limitations to the study: This study is retrospective, monocentric and therefore subject to demographic bias. The predominance of women in all the groups was particularly intriguing and not necessarily representative of common SpA patients. This may be explained by the fact that, in Switzerland, introduction of a biologic therapy does not need to done in hospitals but can be initiated by rheumatologists working in private practice. Therefore, in our tertiary center, we tend to select atypical SpA patients, in 
particular women. As usual in clinical practice, the overall drug survival rates obtained are a reflection of a clinical decision by the treating physician and the validity of the results can be unceasingly questioned. We therefore cannot exclude that some of our results; in particular, the relatively low retention rate in comparison to the literature may be due to local behavior in anti-TNF use. Moreover, the sizes of the different groups were relatively small, limiting statistical power. We suspect that with larger patient groups the trend to shorter drug survival in the ASAS non-classified group would become statistically significant and clinically relevant. Finally, we lacked some data to calculate relevant correlation between failures, disease activity measures and treatment withdrawals.

In conclusion, results from this study suggest that antiTNF drug survival is relatively independent of the classification criteria at least in this "real-life" monocentric selected patient cohort. The study also provides some insights into the characteristics of ASAS classified/nonclassified patients. It highlights some of the pitfalls of SpA classifications; in particular, the difficulty in the diagnosis of sacroiliitis on conventional radiographs and MRI. Among the predictors of good response to biologic therapy proposed in the literature: elevated CRP, positive MRIs for inflammation around sacroiliac joints; we found elevated CRP to be significant only. Results should be confirmed in larger multicentric cohorts.

\section{References}

1. Sieper J (2009) Developments in the scientific and clinical understanding of the spondyloarthritides. Arthritis Res Ther 11(1):208. doi: $10.1186 /$ ar2562

2. Rudwaleit M, van der Heijde D, Landewe R, Listing J, Akkoc N, Brandt J, Braun J, Chou CT, Collantes-Estevez E, Dougados M, Huang F, Gu J, Khan MA, Kirazli Y, Maksymowych WP, Mielants H, Sorensen IJ, Ozgocmen S, Roussou E, Valle-Onate R, Weber U, Wei J, Sieper J (2009) The development of Assessment of SpondyloArthritis international Society classification criteria for axial spondyloarthritis (part II): validation and final selection. Ann Rheum Dis 68(6):777-783. doi:10.1136/ard.2009.108233

3. Sieper J (2011) Spondyloarthropathies in 2010: new insights into therapy-TNF blockade and beyond. Nat Rev Rheumatol 7(2):7880. doi:10.1038/nrrheum.2010.224

4. van der Heijde D, Sieper J, Maksymowych WP, Dougados M, Burgos-Vargas R, Landewe R, Rudwaleit M, Braun J, Assessment of SpondyloArthritis international S (2011) 2010 Update of the international ASAS recommendations for the use of anti-TNF agents in patients with axial spondyloarthritis. Ann Rheum Dis 70(6):905-908. doi:10.1136/ard.2011.151563

5. Goh L, Samanta A (2009) A systematic MEDLINE analysis of therapeutic approaches in ankylosing spondylitis. Rheumatol Int 29(10):1123-1135. doi:10.1007/s00296-009-0973-9

6. Dougados M, Simon P, Braun J, Burgos-Vargas R, Maksymowych WP, Sieper J, van der Heijde D (2011) ASAS recommendations for collecting, analysing and reporting NSAID intake in clinical trials/epidemiological studies in axial spondyloarthritis. Ann Rheum Dis 70(2):249-251. doi:10.1136/ard.2010.133488
7. Sieper J, van der Heijde D, Dougados M, Brown LS, Lavie F, Pangan AL (2012) Early response to adalimumab predicts longterm remission through 5 years of treatment in patients with ankylosing spondylitis. Ann Rheum Dis 71(5):700-706. doi:10.1 136/annrheumdis-2011-200358

8. Gerard S, le Goff B, Maugars Y, Berthelot JM (2008) Six-month response to anti-TNF drugs in axial spondylarthropathy according to the fulfillment or not of New-York criteria for ankylosing spondylitis or French recommendations for anti-TNF use. A "real life" retrospective study on 175 patients. Joint Bone Spine 75(6):680-687. doi:10.1016/j.jbspin.2008.03.006

9. Ciurea A, Scherer A, Exer P, Bernhard J, Dudler J, Beyeler B, Kissling R, Stekhoven D, Rufibach K, Tamborrini G, Weiss B, Mueller R, Nissen MJ, Michel BA, van der Heijde D, Dougados M, Boonen A, Weber U, Rheumatologists of the Swiss Clinical Quality Management Program for Axial S (2013) Tumor necrosis factor-alpha inhibition in radiographic and non-radiographic axial spondyloarthritis: results from a large observational cohort. Arthritis Rheum. doi:10.1002/art.38140

10. Du Pan SM, Dehler S, Ciurea A, Ziswiler HR, Gabay C, Finckh A, Swiss Clinical Quality Management P (2009) Comparison of drug retention rates and causes of drug discontinuation between anti-tumor necrosis factor agents in rheumatoid arthritis. Arthritis Rheum 61(5):560-568. doi:10.1002/art.24463

11. Glintborg B, Ostergaard M, Krogh NS, Tarp U, Manilo N, Loft AG, Hansen A, Schlemmer A, Fana V, Lindegaard HM, Nordin H, Rasmussen C, Ejstrup L, Jensen DV, Petersen PM, Hetland ML (2013) Clinical response, drug survival and predictors thereof in 432 ankylosing spondylitis patients after switching tumour necrosis factor alpha inhibitor therapy: results from the Danish nationwide DANBIO registry. Ann Rheum Dis 72(7):1149-1155. doi:10.1136/annrheumdis-2012-201933

12. Sieper J, van der Heijde D, Landewe R, Brandt J, BurgosVagas R, Collantes-Estevez E, Dijkmans B, Dougados M, Khan MA, Leirisalo-Repo M, van der Linden S, Maksymowych WP, Mielants H, Olivieri I, Rudwaleit M (2009) New criteria for inflammatory back pain in patients with chronic back pain: a real patient exercise by experts from the Assessment of SpondyloArthritis international Society (ASAS). Ann Rheum Dis 68(6):784788. doi:10.1136/ard.2008.101501

13. Calin A (1987) Diagnostic criteria (new and old) for the spondylarthropathies. Clin Exp Rheumatol 5(2):101-102

14. Rudwaleit M, Jurik AG, Hermann KG, Landewe R, van der Heijde D, Baraliakos X, Marzo-Ortega H, Ostergaard M, Braun J, Sieper J (2009) Defining active sacroiliitis on magnetic resonance imaging (MRI) for classification of axial spondyloarthritis: a consensual approach by the ASAS/OMERACT MRI group. Ann Rheum Dis 68(10):1520-1527. doi:10.1136/ard.2009.110767

15. Luc M, Gossec L, Ruyssen-Witrand A, Salliot C, Duclos M, Guignard S, Dougados M (2007) C-reactive protein predicts tumor necrosis factor-alpha blocker retention rate in axial ankylosing spondylitis. J Rheumatol 34(10):2078-2081

16. Rudwaleit M, Schwarzlose S, Hilgert ES, Listing J, Braun J, Sieper J (2008) MRI in predicting a major clinical response to antitumour necrosis factor treatment in ankylosing spondylitis. Ann Rheum Dis 67(9):1276-1281. doi:10.1136/ard.2007.073098

17. Poddubnyy D, Haibel H, Listing J, Marker-Hermann E, Zeidler H, Braun J, Sieper J, Rudwaleit M (2012) Baseline radiographic damage, elevated acute-phase reactant levels, and cigarette smoking status predict spinal radiographic progression in early axial spondylarthritis. Arthritis Rheum 64(5):1388-1398. doi:10.1002/ art.33465

18. Kristensen LE, Karlsson JA, Englund M, Petersson IF, Saxne T, Geborek P (2010) Presence of peripheral arthritis and male sex predicting continuation of anti-tumor necrosis factor therapy in ankylosing spondylitis: an observational prospective cohort study 
from the South Swedish Arthritis Treatment Group Register. Arthritis Care Res 62(10):1362-1369. doi:10.1002/acr.20258

19. Bereau M, Prati C, Wendling D (2011) Sacroiliac edema by MRI does not always indicate spondylarthritis. Joint Bone Spine 78(6):646. doi:10.1016/j.jbspin.2011.05.011

20. Poddubnyy D, Brandt H, Vahldiek J, Spiller I, Song IH, Rudwaleit M, Sieper J (2012) The frequency of non-radiographic axial spondyloarthritis in relation to symptom duration in patients referred because of chronic back pain: results from the Berlin early spondyloarthritis clinic. Ann Rheum Dis 71(12):19982001. doi:10.1136/annrheumdis-2012-201945 